\title{
Análise de risco da ocorrência de Sigatoka-negra baseada em modelos polinomiais: um estudo de caso
}

\author{
Hugo do Nascimento Bendini ${ }^{1,3}$, Wilson da S. Moraes ${ }^{2}$, Silvia H.M.G. da Silva ${ }^{2}$, Erika S. Tezuka ${ }^{1,3}$ \& Paulo \\ Estevão Cruvinel ${ }^{1,3}$
}

'Embrapa Instrumentação, 13560-970, São Carlos, SP, Brasil; '2Universidade Estadual Paulista "Júlio de Mesquita Filho" (UNESP), Campus Experimental de Registro, Rua Nelson Brihi Badur, 430, 11900-000, Registro, SP, Brasil; ${ }^{3}$ Universidade Federal de São Carlos, Departamento de Computação, 13565-905, São Carlos, SP, Brasil

Autor para correspondência: Hugo do Nascimento Bendini, e-mail: hnbendini@gmail.com

\section{RESUMO}

A Sigatoka-negra (Mycosphaerella fijiensis) ameaça os bananais comerciais em todas as áreas produtoras do mundo e provoca danos quantitativos e qualitativos na produção, acarretando sérios prejuízos financeiros. Faz-se necessário o estudo da vulnerabilidade das plantas em diversos estádios de desenvolvimento e das condições climáticas favoráveis à ocorrência da doença. Objetivou-se com este trabalho desenvolver um modelo probabilístico baseado em funções polinomiais que represente o risco de ocorrência da Sigatokanegra em função da vulnerabilidade decorrente de fatores intrínsecos à planta e ao ambiente. Realizou-se um estudo de caso, em bananal comercial localizado em Jacupiranga, Vale do Ribeira, SP, considerando o monitoramento semanal do estado da evolução da doença, séries temporais de dados meteorológicos e dados de sensoriamento remoto. Foram gerados mapas georreferenciados do risco da Sigatoka-negra em diferentes épocas do ano. Um modelo para estimar a evolução da doença a partir de imagens de satélite foi obtido com coeficiente de determinação $\mathrm{R}^{2}$ igual a 0,9 . A metodologia foi desenvolvida para a detecção de épocas e locais que reúnem condições favoráveis à ocorrência da Sigatoka-negra e pode ser aplicada, com os devidos ajustes, em diferentes localidades, para avaliar o risco da ocorrência da doença em polos produtores de banana.

Palavras-chave: Mychosphaerella fijiensis, modelagem, previsão.

\begin{abstract}
Risk analysis of Black Sigatoka occurrence based on polynomial models: A case study

Black Sigatoka (Mycosphaerella fijiensis) threatens the banana trade in most of world's producing areas, damaging the production and bringing significant financial loss. It is necessary to study the plant's susceptibility in the various developmental stages and climatic conditions that favor the disease occurrence. This paper presents a probabilistic model based on polynomial functions for estimating the risk of Black Sigatoka occurrence. A case study was developed in a commercial banana plantation located in Jacupiranga, Vale do Ribeira, SP, Brazil, considering the weekly monitoring of the disease's state evolution (EE), time series of meteorological data and remote sensing data. Georeferenced risk maps were prepared for different dates. We obtained a model to estimate the evolution of the disease from satellite imagery, with a coefficient of determination of 0.9 . This methodology was developed for the detection of times and locations that have favorable conditions to the occurrence of Black Sigatoka and can be applied, with appropriate adjustments for different locations, to assess the risk of disease occurrence in other areas of banana production.
\end{abstract}

Key words: Mychosphaerella fijiensis, forecasting, modeling.

\section{INTRODUÇÃO}

A banana é a fruta mais consumida no mundo (FAO, 2007) e considerada um alimento básico em diversos países. O Brasil é o quarto maior produtor mundial (FAO, 2008) e os principais polos produtores estão no Vale do Ribeira, litoral Sul do Estado de São Paulo (23 municípios); Norte de Minas Gerais (Janaúba, Jaíba, Pirapora, Montes Claros e Itacarambi); Norte de Santa Catarina (Corupá, Massaranduba, Jaraguá do Sul, Guaramirim, Praia Grande, Luis Alves e Schroeder); Nordeste do Brasil (Petrolina, Juazeiro, Bom Jesus da Lapa e Formoso); e no Espírito Santo (Matthiesen \& Boteon, 2002).
A cultura está presente na maioria dos estados brasileiros e a cadeia produtiva é composta principalmente por pequenos produtores. O estado de São Paulo é o segundo maior produtor de banana do Brasil, sendo a região do Vale do Ribeira responsável por cerca de $80 \%$ da produção estadual; nessa região, cerca de $80 \%$ dos produtores são pequenos agricultores (Moraes et al., 2006). O clima da região é caracterizado por alta umidade relativa (60 a $100 \%$ ) durante todos os meses do ano, com um dos maiores índices pluviométricos do país (1500 a $2000 \mathrm{~mm}$ anuais) (Fukuda et al., 2007), o que torna as condições altamente favoráveis ao desenvolvimento da bananeira, mas também para a Sigatoka-negra, considerada a doença mais severa 
e destrutiva da cultura. A Sigatoka-negra é causada pelo fungo Mycosphaerella fijiensis Morelet [fase assexuada Paracercospora fijiensis (Morelet)]. O primeiro registro desta doença no Brasil deu-se na fronteira com a Colômbia e o Peru, em 1998, mas a disseminação foi rápida para todos os estados da região Amazônica (Pereira et al., 2000; Gasparotto et al., 2006). A doença chegou à região Sudeste em junho de 2004, em Miracatu (SP) e, concomitantemente, em todos os municípios produtores de banana do Estado (Ferrari \& Nogueira, 2003).

O fato de a Sigatoka-negra ser uma doença rápida e destrutiva tem gerado grandes preocupações entre os produtores, técnicos e pesquisadores, principalmente, quando a bananicultura é a principal atividade econômica da região. Por isso, as áreas consideradas livres da doença buscam a todo custo impedir a sua introdução, o que implicaria em aumento dos custos de proteção e de produção, e em perda de um importante diferencial na busca de abertura do mercado externo.

O controle da doença em cultivares suscetíveis, como as do subgrupo Prata e Cavendish (Nanica e Nanicão), tem sido feito por meio de pulverizações alternadas de fungicidas sistêmicos e protetores, com intervalos definidos pelo monitoramento semanal da severidade da doença. O monitoramento da severidade da Sigatoka-negra tem sido realizado pelo método de Estado da Evolução (EE), descrito por Fouré (1988) e ajustado por Moraes et al. (2005). Entretanto, é uma tarefa trabalhosa que requer tempo, mão de obra e infraestrutura especializadas.

Geralmente, a decisão do momento da aplicação de fungicida é tomada com base no segundo aumento consecutivo da severidade da doença ou no primeiro aumento superior a 200 pontos, caso ocorram chuvas de pelo menos $100 \mathrm{~mm}$ ou temperaturas mais elevadas nas duas semanas que antecederam à leitura da severidade (Moraes et al., 2005). Essa decisão tomada em função dos dados climáticos do local, além de ser feita de forma subjetiva, não considera a variabilidade espacial na região, pois a estimativa generaliza a área de produção a partir de poucas amostras. A previsão de períodos considerados favoráveis à ocorrência da doença pode auxiliar no seu controle, sendo determinantes o efeito de períodos de molhamento foliar e temperaturas favoráveis (Berger, 1989; Bergamin Filho \& Amorim, 1996).

Para a Sigatoka-negra na região do Vale do Ribeira, Fukuda \& Moraes (2007) determinaram, com base em intervalos semanais, os valores de precipitação acumulada em até $100 \mathrm{~mm}$, temperaturas mínimas inferiores a $18^{\circ} \mathrm{C}$ e máximas inferiores a $28^{\circ} \mathrm{C}$, como sendo desfavoráveis ao desenvolvimento da doença. A umidade relativa média semanal de $84,4 \%$ favorece o desenvolvimento da Sigatokanegra na região amazônica (Acre, 2000). Cronshaw (1984) relacionou o efeito do acúmulo de horas semanais com umidade relativa acima de $90 \%$ com o progresso da doença e verificou que valores superiores a 60 horas são favoráveis a doença; entre 40 e 50 horas são moderadamente favoráveis; entre 10 e 40 horas são ligeiramente favoráveis; e inferiores a 10 horas não são favoráveis ao desenvolvimento da Sigatoka-negra.

Dispondo de uma rede de estações meteorológicas localizadas nas proximidades da região monitorada é possível elaborar mapas de zonas de risco de epidemias para definir um zoneamento agroclimático (Caramori et al., 1994; Pugsley et al., 2001; Cruvinel et al., 2002). O desenvolvimento de mapas de zonas de risco acoplados a modelos de predição pode ser útil para indicar áreas geográficas ou épocas do ano mais favoráveis à ocorrência de epidemias. A partir dessa base de dados, sistemas computacionais podem ser utilizados para verificar se as condições meteorológicas estão favoráveis à ocorrência de infecção pelos patógenos.

Moraes et al. (2006) analisaram o progresso da Sigatoka-negra durante o período de fevereiro a dezembro de 2005, em propriedade do município de Jacupiranga (SP), região do Vale do Ribeira; os autores demonstraram que a severidade da doença foi mais expressiva entre os meses de novembro a março, quando foram observadas temperaturas e precipitações mais elevadas, enquanto que no período menos chuvoso, com temperaturas mais amenas, entre junho a dezembro, a doença foi desfavorecida. Esses autores ainda simularam o comportamento da doença com uma função de regressão, em que as médias de temperatura máxima e mínima e a precipitação acumulada durante uma e duas semanas antes da leitura foram os parâmetros de entrada, tendo sido os que mais se correlacionaram com a severidade da doença com duas semanas de antecedência. Porém, esta simulação foi realizada em um bananal específico de 15 ha com base nos dados de severidade e meteorológicos predominantes no bananal.

O presente trabalho teve por objetivo desenvolver um método de análise de risco de ocorrência da Sigatoka-negra, tendo como referência o trabalho desenvolvido por Moraes et al. (2006), por meio da integração da probabilidade de ocorrência de fatores climáticos favoráveis à doença, utilizando-se modelos polinomiais e processamento digital de imagens de satélite.

\section{MATERIAL E MÉTODOS}

O estudo foi desenvolvido em uma propriedade de 15 ha de bananeira Nanica, cultivar Grand Naine, de oito anos de produção, localizada no município de Jacupiranga, no Vale do Ribeira, Estado de São Paulo ( $24^{\circ} 52^{\prime}$ 53,8" $\mathrm{S}$ e $\left.48^{\circ} 06^{\prime} 22,1^{\prime \prime} \mathrm{O}\right)$. Nessa propriedade, a severidade da Sigatoka-negra foi monitorada semanalmente, durante o período de fevereiro a dezembro de 2005, pelo método de Estado de Evolução (EE), descrito por Fouré (1988) e ajustado por Moraes et al. (2005). Esse método considera o ritmo de emissão foliar semanal (REFx) e a severidade da doença (Sev) na segunda, terceira e quarta folha, a partir da folha mais nova da planta ou folha "vela", em dez plantas amostradas em até 50 ha, e é definido pela Equação 1: 


$$
E E=S e v \cdot R E F x \quad(1)
$$

A severidade da doença foi determinada com base na qualificação e quantificação dos estádios precoces do desenvolvimento dos sintomas da doença (1 - ponto; 2 traço; e 3 - estria), observados na face abaxial da segunda, terceira e quarta folha das dez plantas amostradas. $\mathrm{O}$ estado da evolução foi expresso pelo produto do somatório da pontuação obtida com base nos coeficientes de gravidade (Tabela 1), a partir da quantificação do estádio precoce mais evoluído do desenvolvimento dos sintomas, observado nas folhas amostradas de cada planta, pelo ritmo de emissão foliar ponderado entre as dez plantas.

TABELA 1 - Pontuação ou coeficiente de gravidade da Sigatokanegra em função da folha afetada, em que o sinal - ou + significa menos ou mais de 50 unidades do estádio mais evoluído do desenvolvimento dos sintomas da doença

\begin{tabular}{cccc}
\hline \hline \multirow{2}{*}{ Estádio } & \multicolumn{3}{c}{ Número da folha } \\
\cline { 2 - 4 } & II & III & IV \\
\hline-1 & 60 & 40 & 20 \\
+1 & 80 & 60 & 40 \\
-2 & 100 & 80 & 60 \\
+2 & 120 & 100 & 80 \\
-3 & 140 & 120 & 100 \\
+3 & 160 & 140 & 120 \\
\hline
\end{tabular}

Séries temporais de variáveis meteorológicas disponíveis na estação meteorológica do Centro Integrado de Informações Agrometeorológicas (CIIAGRO), no município de Jacupiranga (SP) e na estação de superfície convencional do Instituto Nacional de Meteorologia (INMET), no município de Iguape (SP) foram utilizadas no presente estudo. As variáveis estudadas foram a temperatura máxima (Tmax) e mínima (Tmin), precipitação acumulada (Pp), umidade relativa (Ur) e duração do período de molhamento foliar (DPM). Para as duas últimas variáveis foram obtidos os dados horários. Na etapa dedicada ao tratamento das séries temporais, os dados faltantes foram corrigidos por interpolação linear. Os dados relativos à duração do período de molhamento foliar (DPM) foram determinados de acordo com a metodologia descrita por Gasparotto (1988), em que o DPM é igual ao somatório do número de horas com umidade relativa superior a 90\%.

Para analisar o desenvolvimento da doença no campo, a partir do sensoriamento remoto, foram utilizados dados de reflectância observados na região do verde $(0,52$ $\mu \mathrm{m}$ a $0,6 \mu \mathrm{m}$ ), obtidos em imagens da banda 2 do sensor TM LANDSAT-5, sob resolução espacial de $30 \mathrm{~m}$, referentes às órbitas-ponto 220/77, entre fevereiro e dezembro de 2005, totalizando seis imagens. As imagens foram obtidas no catálogo do satélite do Instituto Nacional de Pesquisas Espaciais (INPE). As operações com as imagens foram realizadas no Sistema de Processamento de Informações Georeferenciadas (SPRING), versão 5.0.6, desenvolvido pelo INPE (Câmara et al., 1996).

Inicialmente, procedeu-se o registro das imagens pelo método imagem-imagem (Brown, 1992), que corrige a geometria da imagem a partir de pontos conhecidos, utilizando-se como referência uma imagem ortorretificada na projeção UTM e Datum WGS-84, seguido da ampliação linear de contraste para todas as demais imagens. Dessa forma, localizou-se a área em estudo, nas imagens, pelas coordenadas UTM, obtidas em condições de campo, e procedeu-se a extração dos valores de níveis de cinza (NC), utilizando-se uma janela de amostragem de 3 x 3 pixels, cujo pixel central correspondeu à área onde a severidade da Sigatoka-negra foi monitorada.

Para concepção do modelo, as séries temporais dos dados climáticos foram estudadas, para a determinação de 10 classes de intervalos de dados equidistantes, baseados na amplitude dos valores para cada variável. Dessa forma, as frequências relativas foram calculadas para cada classe, ou seja, a probabilidade de ocorrência de cada uma e, posteriormente, submetidas à análise de regressão polinomial para o ajuste da curva, como polinômios de diferentes ordens. Posteriormente, os modelos definidos para cada variável foram selecionados pelo coeficiente de determinação $\left(\mathrm{R}^{2}\right)$ e o erro padrão $(\varepsilon)$.

A partir dos valores de probabilidade de ocorrência das classes ajustados, realizou-se a normalização entre 0 e 1 , com valores máximos e mínimos, utilizando-se a Equação 2: em que $X_{i}$ é o valor a ser normalizado; $i$, neste caso, varia de 1 a 10 , e $\max$ e min são os valores máximos e mínimos, respectivamente.

$$
X_{n}=\frac{X_{i}-\min }{\max -\min }
$$

A partir do conhecimento teórico e prático sobre a doença, os intervalos de classes de variáveis foram definidos em três níveis de favorabilidade à ocorrência da Sigatokanegra, alto, médio e baixo (Tabela 2).

No caso dos valores de NC da imagem LANDSAT-5, aplicou-se previamente uma regressão polinomial com os valores de EE correspondentes à data da leitura com diferença de no máximo dois dias em relação à data de coleta da imagem, a fim de obter uma equação que relacionasse o valor de $\mathrm{NC}$ com a severidade da doença. Assim, níveis de favorabilidade foram atribuídos de acordo com o comportamento da curva de severidade da doença, observada no gráfico, ou seja, a partir de que valores de NC os valores de EE apresentaram tendência de aumentar ou diminuir. Feito isso, procedeu-se à normalização dos valores de probabilidades de ocorrência das classes dentro de cada nível de favorabilidade, utilizando-se a Equação 2. Nesse caso, os valores máximos e mínimos foram obtidos dentro de cada nível de favorabilidade. Desse modo, a escala de risco pôde ser representada da seguinte forma: 


$$
\begin{array}{ll}
\text { i. } & 0,00 \leq \text { baixo } \leq 0,33 \\
\text { ii. } & 0,34 \leq \text { médio } \leq 0,67 \\
\text { iii. } & 0,68 \leq \text { alto } \leq 1,00
\end{array}
$$

Para cálculo do risco, utilizou-se a metodologia proposta por Cruvinel et al. (2006), em que as probabilidades normalizadas para cada variável foram plotadas nos eixos de uma circunferência de raio unitário. $\mathrm{O}$ risco total correspondeu à área da figura poligonal formada pela união dos vértices, sendo determinada pela soma das áreas dos triângulos formados dentro da figura (Figura 1). Uma vez conhecido um dos ângulos e os dois lados de cada triângulo, a área total da figura pôde ser determinada pela Equação 3:

$$
a_{T}=\sum_{i=1}^{6} \frac{a_{i} b_{i} \operatorname{sen} \alpha_{i}}{2}
$$

em que, $a_{T}$ é área total da figura, $a$ e $b$, são os lados conhecidos dos triângulos, e $\alpha$ é o ângulo formado entre os vetores $\left(60^{\circ}\right)$.

O risco total de ocorrência da Sigatoka-negra é, portanto, a relação de intersecção entre as probabilidades normalizadas de ocorrência da respectiva classe de cada variável (Equação 4).

$P($ Risco $)=P(T$ max $) \cap P(T$ min $) \cap P(P p) \cap P(U r) \cap P(D P M) \cap P(N C)$

Assim, figuras de mérito foram geradas para cada data correspondente às imagens de satélite, sendo que para a variável $\mathrm{NC}$, dez diferentes valores de probabilidade foram obtidos em função da classe de intervalo em que se encontrou o valor do pixel correspondente, permitindo a espacialização do risco. Desta forma, os diferentes valores de NC encontrados permitiram inferir sobre a intensidade da doença em determinado pixel, ou seja, região que contribui para aumentar o risco da doença; uma vez que a Sigatokanegra é uma doença policíclica, cada planta doente contribui para intensificar a disseminação da doença.

Para geração do mapa de risco, as matrizes de valores de $\mathrm{NC}$ dos pixels foram extraídas das imagens de satélite da propriedade em estudo e, para cada data em questão, cada pixel foi substituído pelo valor de risco obtido pela respectiva figura de mérito. Assim, uma ferramenta desenvolvida em Delphi foi utilizada para mapear os pixels em uma escala de cor, em que verde representa o risco baixo, amarelo o risco médio e vermelho o risco alto, para a ocorrência da Sigatoka-negra. Finalmente, a matriz gerada foi adaptada à base georreferenciada no SPRING, obtendose, assim, os mapas.

\section{RESULTADOS E DISCUSSÃO}

A Figura 2 ilustra as figuras de méritos para as diferentes datas avaliadas, sendo a variável NC avaliada em apenas uma situação, ou seja, com valor máximo igual a 1. A Figura 3 apresenta os gráficos de distribuições de probabilidades selecionadas para cada variável do sistema, bem como seus respectivos coeficientes de determinação $\left(\mathrm{R}^{2}\right)$.

A utilização de modelos polinomiais, em abordagem probabilística, descreve melhor o fenômeno estudado, uma vez que os dados variam de forma não linear em um sistema dinâmico e complexo, como é a natureza. Tal fato pode ser verificado pelos altos valores dos coeficientes de determinação $\left(\mathrm{R}^{2}\right)$, com exceção para as variáveis Ur e DPM, cujos valores foram inferiores a 0,8 , o que pode estar relacionado ao fato de que foram obtidos na frequência horária e que houve ausência de $12,08 \%$ dos dados relativos à série temporal para Ur, sendo necessária a interpolação dos valores. Tais modelos foram elaborados especificamente para a região em estudo, mas a metodologia pode ser aplicada em diferentes regiões produtoras de banana, utilizando-se outros níveis semi-arbitrários de favorabilidade à doença, bem como imagens de satélite e séries temporais dos dados meteorológicos para cada região em questão.

$\mathrm{O}$ peso em que cada variável contribuiu para aumentar ou diminuir o risco não foi analisado, pois o desenvolvimento do patógeno causador da Sigatokanegra está diretamente relacionado ao efeito conjunto das variáveis, conforme os trabalhos desenvolvidos por Moraes

\begin{tabular}{|c|c|c|c|c|c|c|c|c|}
\hline Classes & $\operatorname{Tmax}\left({ }^{\circ} \mathrm{C}\right)$ & Nível & $\operatorname{Tmin}\left({ }^{\circ} \mathrm{C}\right)$ & Nível & Pp (mm) & Nível & DPM (h) & Nível \\
\hline 1 & $19,13-20,81$ & Baixo & $7,57-9,08$ & Baixo & $0,00-20,09$ & Baixo & $77,51-79,00$ & Baixo \\
\hline 2 & $20,82-22,50$ & Médio & $9,09-10,6$ & Baixo & $20,10-40,19$ & Baixo & $79,01-80,49$ & Baixo \\
\hline 3 & $22,51-24,19$ & Médio & $10,61-12,12$ & Baixo & $40,20-60,29$ & Médio & $80,50-81,99$ & Médio \\
\hline 4 & $24,20-25,88$ & Alto & $12,13-13,64$ & Baixo & $60,30-80,39$ & Médio & $82,00-83,48$ & Médio \\
\hline 5 & $25,89-27,58$ & Alto & $13,65-15,16$ & Médio & $80,40-100,49$ & Médio & $83,49-84,98$ & Médio \\
\hline 6 & $27,59-29,27$ & Alto & $15,17-16,68$ & Médio & $100,50-120,59$ & Alto & $84,99-86,47$ & Alto \\
\hline 7 & $29,28-30,96$ & Alto & $16,69-18,20$ & Médio & $120,60-140,69$ & Alto & $86,48-87,96$ & Alto \\
\hline 8 & $30,97-32,65$ & Médio & $18,21-19,72$ & Alto & $140,70-160,79$ & Alto & $87,97-89,46$ & Alto \\
\hline 9 & $32,66-34,34$ & Médio & $19,73-21,24$ & Alto & $160,80-180,89$ & Alto & $89,47-90,95$ & Alto \\
\hline 10 & $34,35-36,03$ & Baixo & $21,25-22,76$ & Alto & $180,90-200,99$ & Médio & $90,96-92,44$ & Alto \\
\hline
\end{tabular}
et al. (2006) e Fukuda \& Moraes (2007). Estes autores

TABELA 2 - Intervalos de classes das variáveis e respectivos níveis de favorabilidade à Sigatoka-negra, utilizados com base em critério semi-arbitrário

Tmax = temperatura máxima; Tmin = temperatura mínima; $\mathrm{Pp}=$ precipitação acumulada; $\mathrm{DPM}=$ duração do período de molhamento foliar. 


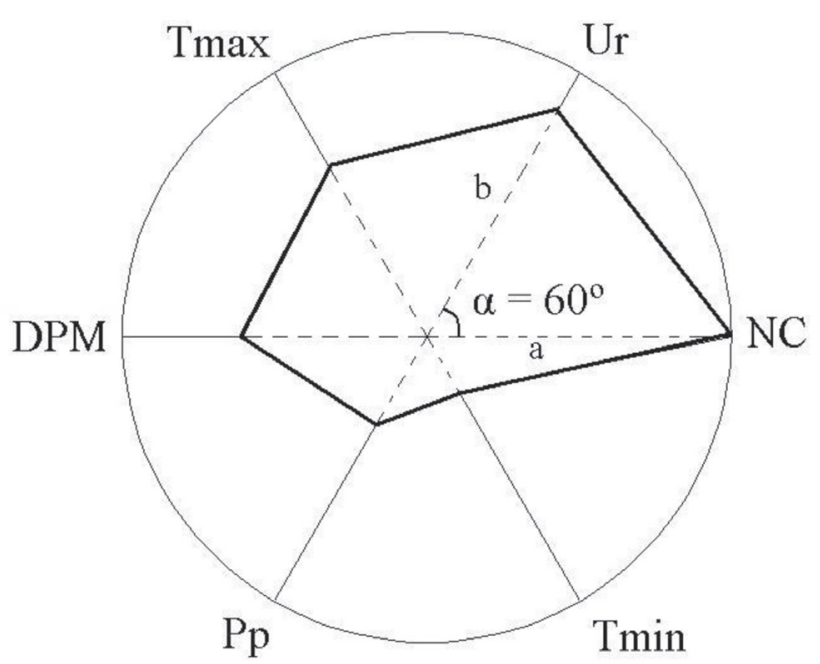

FIGURA 1 - Figura de mérito para cálculo da área integrada, em que cada eixo representa a probabilidade normalizada para cada variável e a área total corresponde ao risco de ocorrência da Sigatoka-negra. Tmax = temperatura máxima; $T \min =$ temperatura mínima; Pp = precipitação acumulada; DPM = duração do período de molhamento foliar; $\mathrm{Ur}=$ umidade relativa; $\mathrm{NC}=$ níveis de cinza. estudaram a influencia de variáveis climáticas sobre a severidade semanal da Sigatoka-negra na bananeira Nanica cultivar Grand Naine, ao longo de dois anos consecutivos. Análises de correlação e regressão múltiplas, utilizando-se Redes Neurais Artificiais, permitiram estimar a severidade da Sigatoka-negra com duas semanas de antecedência, sendo definida, em 2006, pelo modelo: $E E=-1265+0,5886 p p$ $+73,7879$ tmax $+52,8995$ tmin $\left(\mathrm{R}^{2}=0,90\right)$ e, em 2007, pelo modelo: $\quad E E=-1582,1081+0,6146^{*} P P+31,8881 *$ Tmax $68,3967 *$ Tmin $+147,2701 *$ Tmed $\left(\mathrm{R}^{2}=0,84\right)$. A partir destes resultados propôs-se estudar modelos probabilísticos baseados em funções polinomiais que represente o risco de ocorrência da Sigatoka-negra em função da vulnerabilidade decorrente destas e outras variáveis climáticas.

A Figura 4 ilustra o gráfico do modelo que correlaciona o NC da imagem de satélite com a incidência da doença no campo. O polinômio de terceira ordem foi selecionado em função do seu maior coeficiente de determinação $\left(\mathrm{R}^{2}=0,90\right)$. Tal resultado indicou que a utilização da reflectância dentro do comprimento de onda na faixa do visível, correspondente ao verde, no caso, foi mais adequada.

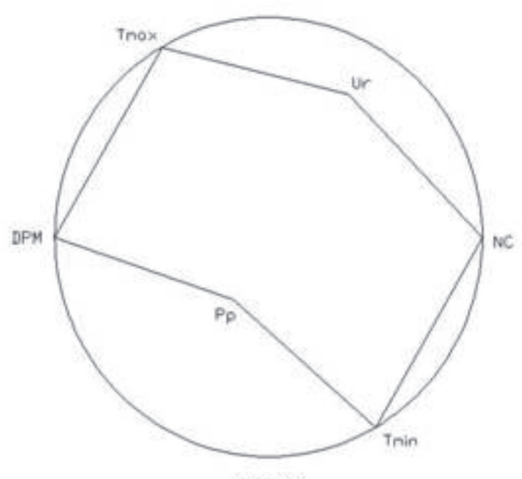

$22 / 02$

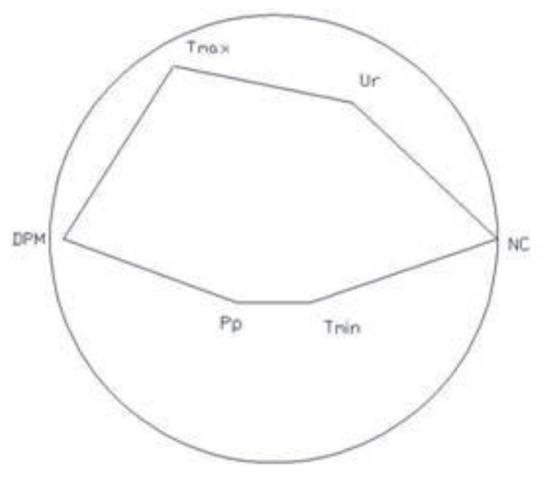

$30 / 06$

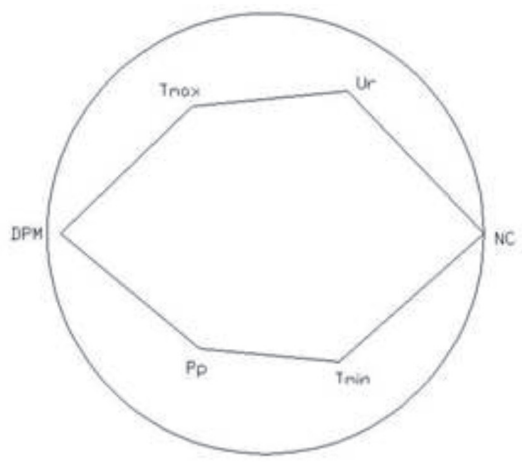

$13 / 05$

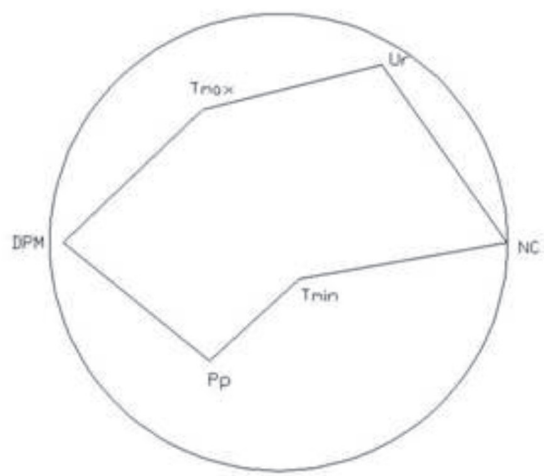

$01 / 08$

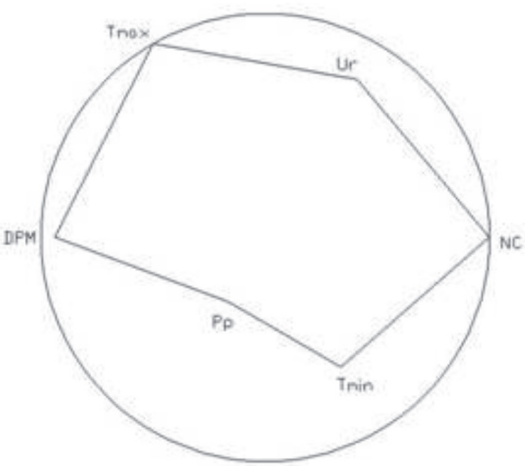

$17 / 06$

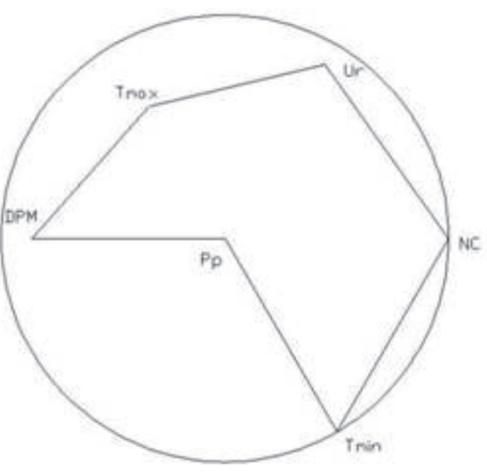

$17 / 08$

FIGURA 2 - Figuras de méritos para as diferentes datas, expressando os valores de probabilidades para cada variável. 


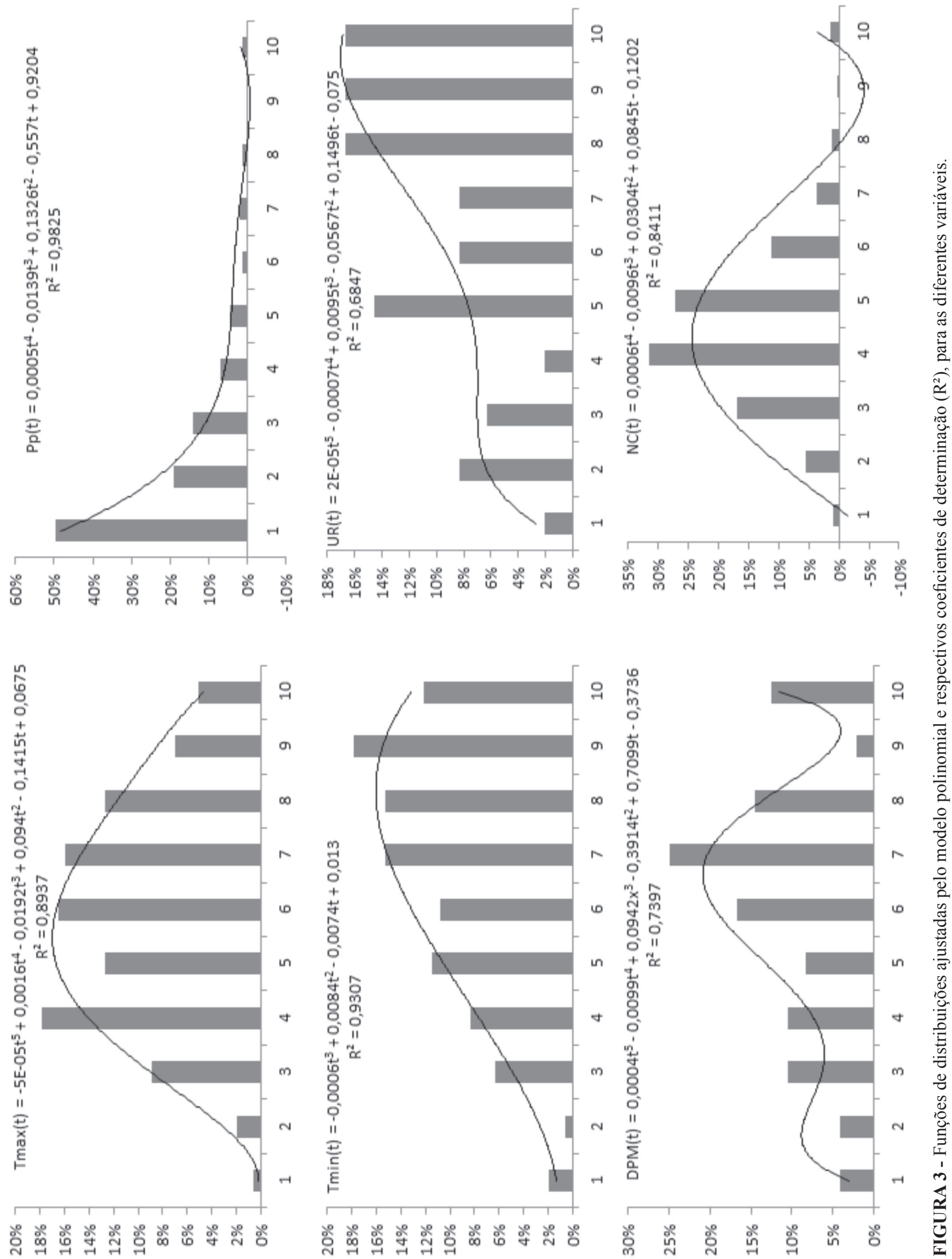


Resultados semelhantes foram encontrados por Lippert et al. (2009), utilizando espectrorradiometria para avaliar o comportamento espectral da espécie Eucalyptus camaldulensis infectada por Phaeophleospora eucalypti, considerando diferentes níveis de dano na área foliar. Neste patossistema, os autores observaram que a região do espectro do visível foi a que melhor detectou a ocorrência do patógeno nos diferentes níveis de danos testados. A utilização de imagens de sensoriamento remoto permitiu espacializar o risco de ocorrência da doença em função do pixel, o que tornou possível realizar inferências em pequenas propriedades, diferentemente do trabalho desenvolvido por Ghini et al. (2007), em que os autores fizeram uma projeção de cenários do comportamento da Sigatoka-negra em função das mudanças climáticas e considerando áreas continentais.

A Figura 5 ilustra os mapas de risco de ocorrência da Sigatoka-negra, obtidos paraas diferentes datas consideradas. Observa-se, pela Tabela 3, que no mapa referente a 22/02
(Figura 5a), época de temperaturas mais elevadas e altas precipitações, $81 \%$ dos pixels foram classificados como de risco alto e, apenas $19 \%$ como de risco médio. $\mathrm{Na}$ época menos chuvosa e com temperaturas mais amenas, caracterizada pelo mapa do dia 13/05 (Figura 5b), 12\% dos pixels foram classificados como de risco baixo e $88 \%$ como de risco médio. Nas demais datas que se concentraram em períodos em que, naquele ano, foram caracterizados por baixas temperaturas e altas umidades relativas, os mapas (Figura 5c) apresentaram-se na totalidade na classe de risco médio.

A metodologia desenvolvida demonstrou-se viável e promissora no que diz respeito à detecção de épocas e locais com condições favoráveis a ocorrência da Sigatoka-negra, para a região considerada. Desta forma, este modelo poderá servir como subsídio para implementação de sistemas de alertas, programas de controle da doença de forma a otimizar o uso de energia, principalmente reduzindo a utilização de fungicidas químicos, o que reflete um ganho econômico e ambiental.

TABELA 3 - Porcentagens de pixels da área em estudo, classificados em diferentes níveis de risco. Jacupiranga (SP), 2005

\begin{tabular}{ccrrrrrr}
\hline \hline Risco & $\mathbf{2 2 / f e v}$ & $\mathbf{1 3} / \mathbf{m a i}$ & $\mathbf{1 7 / j u n}$ & $\mathbf{3 0 / j u n}$ & $\mathbf{0 1 / \text { ago }}$ & $\mathbf{1 7 / \text { ago }}$ \\
\hline Baixo & $0 \%$ & $12 \%$ & $0 \%$ & $0 \%$ & $0 \%$ & $0 \%$ & $100 \%$ \\
Médio & $19 \%$ & $88 \%$ & $100 \%$ & $100 \%$ & $100 \%$ & $0 \%$ & $0 \%$ \\
Alto & $81 \%{ }^{1}$ & $0 \%$ & $0 \%$ & $0 \%$ & $0 \%$ & $0 \%$ \\
\hline
\end{tabular}

${ }^{1}$ Porcentagem da área classificada em diferentes níveis de risco de ocorrência de Sigatoka-negra (\%).

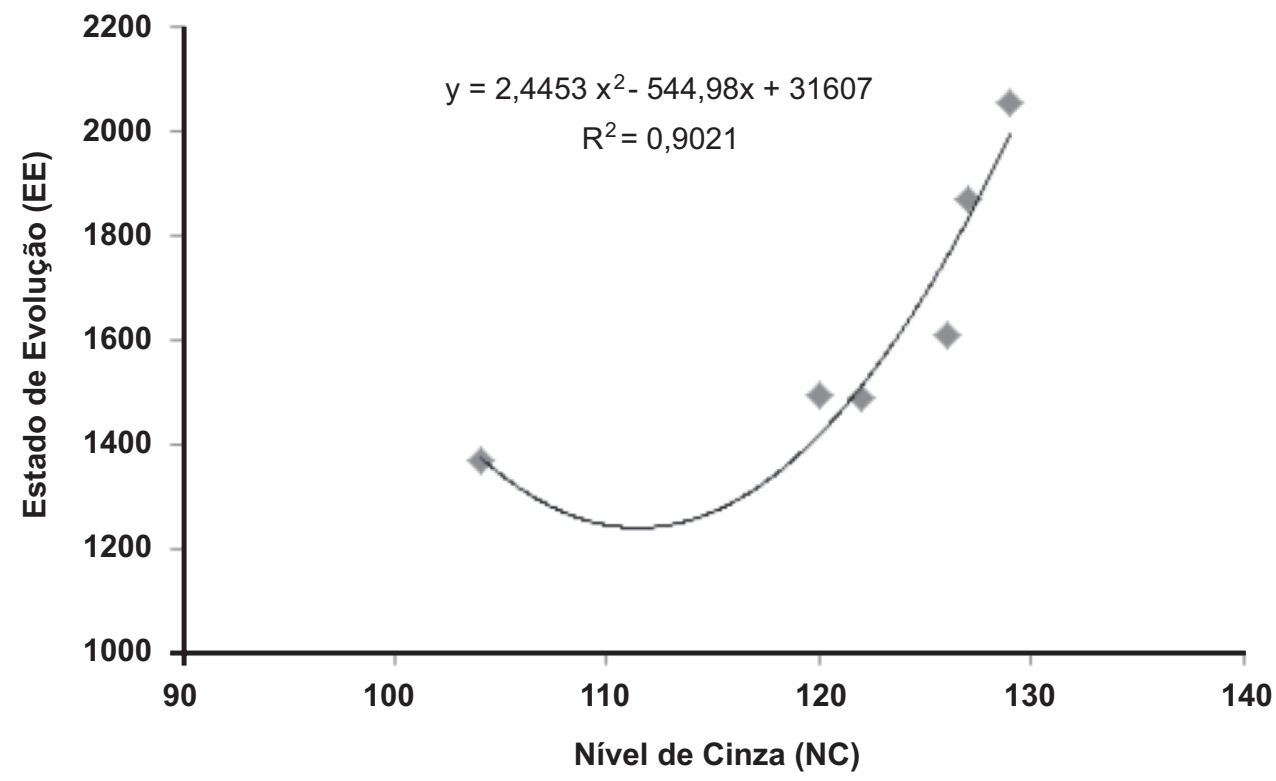

FIGURA 4 - Modelo que correlaciona níveis de cinza (NC) da imagem correspondente a banda 2 do satélite LANDSAT-5, com o estado de evolução ou severidade da doença (EE). 

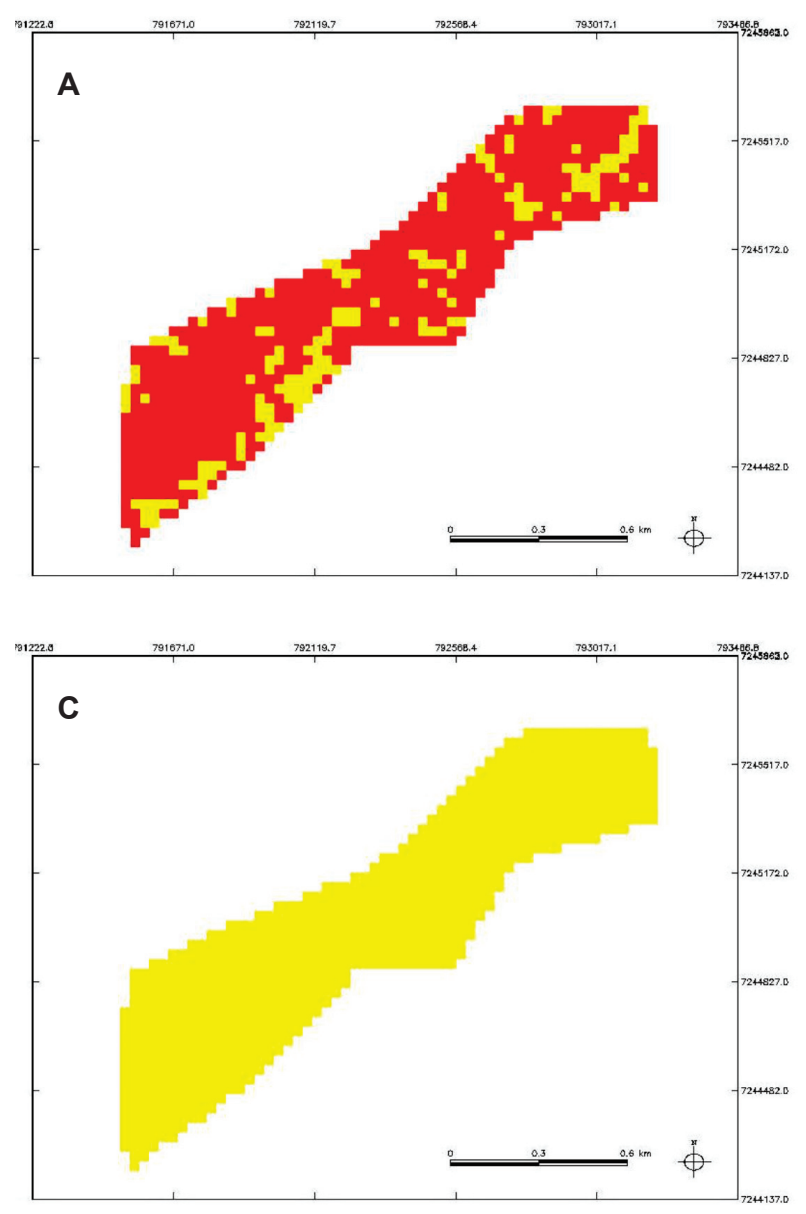

\section{REFERÊNCIAS BIBLIOGRÁFICAS}

Acre (2000) Secretaria de Estado de Ciência, Tecnologia e Meio Ambiente. Programa Estadual de Zoneamento EcológicoEconômico do Estado do Acre. Secretaria de Estado Ciência, Tecnologia e Meio Ambiente.

Bergamin Filho A, Amorim L (1996) Doenças de plantas tropicais: epidemiologia e controle econômico. São Paulo SP. Ed. Agronômica Ceres.

Berger RD (1989) Description and application of some general models for plant disease epidemics. In: Leonard KJ, Fry WE (Eds.) Plant disease epidemiology: Genetics, resistance, and management. New York NY. MacMillan. pp. 15-149.

Brown LG (1992) A survey of image registration techniques. ACM Computing Surveys 24:325-376.

Câmara G, Souza RCM, Freitas UM, Garrido JCP (1996) SPRING: Integrating remote sensing and GIS with object-oriented data modeling. Computers and Graphics 6:13-22.

Caramori PH, Androcioli Filho A, Liberal EG (1994) (Eds.). In: Simpósio Internacional sobre café adensado, Resumos... Londrina PR. IAPAR.

Cronshaw DK (1984) Monitoring of Sigatoka disease

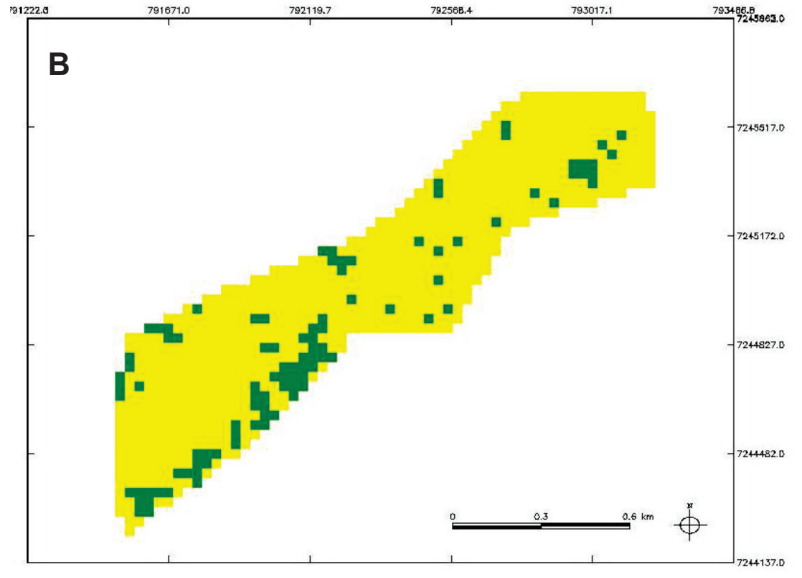

FIGURA 5 - Mapas de risco de ocorrência da Sigatoka-negra para o bananal em estudo em Jacupiranga (SP) em diferentes épocas no ano de 2005: A. 22/02; B. 13/05; e C. 17/06, 30/06, 01/08 e 17/08. Os pixels mapeados em vermelho representam áreas de risco alto; em amarelo, áreas de risco médio e, em verde, áreas de risco baixo.

(Mycosphaerella musicola) in the Windward Islands for tolerance to fungicides. Tropical Pest Management 30:225-229.

Cruvinel PE, Pugsley L, Caramori, PH, Pazinatto FAC (2002) Advanced methodology for zones of risk optimization in agriculture. In: Cruvinel PE, Mascarenhas S (Eds.) Advanced studies in agricultural instrumentation. São Carlos SP. RIMA. pp. 167-205.

Cruvinel PE, Suzumura Filho Y, Mantovani EC (2006) Análise da qualidade da aplicação georeferenciada de chuva artificial em área de plantio com visão computacional e rede neural. In: $35^{\circ}$ Congresso Brasileiro de Engenharia Agrícola - CONBEA, Resumos... João Pessoa PB. SBEA.

Ferrari JT, Nogueira EMC (2003) Como identificar e combater a Sigatoka Negra da bananeira. Fitopatologia Brasileira 28:656663.

Fouré E (1988) Stratégies de lutte contre la cercosporiose noire des bananiers et des plantains provoquée par Mycosphaerella fijiensis Morelet. L'avertissement biologique au Cameroun. Evaluation des possibilités d'amélioration. Fruits 43:269-274.

Fukuda E, Moraes WS (2007) Monitoramento da severidade da Sigatoka Negra em bananais do Vale do Ribeira, São Paulo, Brasil. In: Congresso de Iniciação Científica da UNESP, Resumos... Ilha Solteira SP. UNESP. pp. 22-23. 
Gasparotto L(1988) Epidemiologia do mal das folhas [Mycrocyclus ulei (P. Henn.) V. Arx.] da seringueira (Hevea spp.) Tese DS, Universidade Federal de Viçosa. Viçosa MG.

Gasparotto L, Pereira JCR, Hanada RE, Montarroyos AVV (2006) Sigatoka-negra da bananeira. Manaus AM. Embrapa Amazônia Ocidental.

Ghini R, Hamada E, Gonçalves RRV, Gasparotto L, Pereira JCR (2007) Análise de risco das mudanças climáticas globais sobre a Sigatoka-negra da bananeira no Brasil. Fitopatologia Brasileira 32:197-204.

Lippert DB, Benedetti ACP, Pereira RS, Muniz MFB (2009) Espectrorradiometria de folhas de Eucalyptus camaldulensis Dehnh. atacadas por Phaeophleospora eucalypti. In: XIV Simpósio Brasileiro de Sensoriamento Remoto, Resumos... Natal RN. pp. 2793-2799.

Matthiesen ML, Boteon M (2002) Análise dos principais pólos produtores de banana no Brasil. Piracicaba SP. ESALQ/Hortifruti/ CEPEA.
Moraes WS, Mendonça JC, Fukuda E, Mendes C, Lima JD, Santos AJ (2005) Dominância da Sigatoka-negra em bananais do Vale do Ribeira. Fitopatologia Brasileira 30:193.

Moraes WS, Fukuda E, Mendonça JC, Silva CM, Silva SHM (2006) Behaviour of black Sigatoka in banana plantations of the Ribeira Valley, São Paulo, Brazil. In: XVII Reunião Internacional da Associação para a Cooperação em Pesquisas sobre Banana no Caribe e América Tropical (ACORBAT), Resumos... Joinville SC. IFAC. pp. 656-661.

Pereira JCR, Gasparotto L, Coelho AFS, Véras SM (2000) Doenças da bananeira no Estado do Amazonas. Manaus AM. Embrapa Amazônia Ocidental.

Pugsley L, Cruvinel PE, Caramori PH (2001) New agroclimatic digital images classification system and risk zone mapping. In: $14^{\text {th }}$ Brazilian Symposium on Computer Graphics and Image Processing, Resumos... Florianópolis SC. IEEE Computer Society. pp. 237-244.

TPP 379 - Recebido 17 Agosto 2011 - Aceito 30 Outubro 2012

Editor de Seção: Lilian Amorim 